\title{
Realitätsnahe Bestimmung der Eisenverluste einer elektrischen Maschine mit Einzelzähnen
}

\author{
C. Rollbühler, P. Breining®, J. Kolb, M. Doppelbauer
}

\begin{abstract}
An elektrische Maschinen in elektrifizierten Automobilantrieben werden hohe technische Anforderungen gestellt. Zum einen werden Produktionsmethoden benötigt, welche die Herstellung großer Stückzahlen unter Einhaltung niedriger Kosten ermöglichen. Zum anderen wird eine hohe elektrische Reichweite gefordert, was einen elektrischen Antriebsstrang mit hoher Effizienz und gleichzeitig hoher Leistungsdichte voraussetzt. Grundlegend für die Entwicklung elektrischer Maschinen mit den genannten Eigenschaften sind detaillierte Verlustmodelle. Insbesondere die Modellierung der Ummagnetisierungsverluste, kurz Eisenverluste, im Stator und Rotor der Maschine ist dabei herausfordernd. Neben der Materialwahl und der Maschinengeometrie haben die Fertigungsprozesse einen nicht zu vernachlässigenden Einfluss auf die Verlustcharakteristik der Maschine. In diesem Beitrag wird ein neuer Ansatz zur Bestimmung der Eisenverluste eines einzelnen Statorzahns einer elektrischen Maschine unter Berücksichtigung realitätsnaher Flussbedingungen vorgestellt. Mit dem gezeigten Verfahren können Fertigungseinflüsse auf die Eisenverluste untersucht werden, wobei anwendungsspezifische Geometrie sowie magnetische Ausnutzung des Zahns beibehalten bleiben. Zunächst werden die magnetischen Zustände einer elektrischen Maschine mit Einzelzahnwicklung beschrieben. Nachfolgend wird der Einzelzahn-Prüfstand eingeführt und die Übertragung der relevanten magnetischen Größen der Maschine auf den Prüfstand vorgestellt. Die Bestimmung der Zahnverluste anhand von Messdaten wird gezeigt. Abschließend wird ein Vergleich von Simulation und Messung vorgenommen. Das vorgestellte Verfahren bietet die Möglichkeit, Eisenverlustmodelle an die zusätzlich auftretenden fertigungsbedingten Verluste anzupassen und die Unsicherheiten bei der Verlustmodellierung im Allgemeinen zu verringern.
\end{abstract}

Schlüsselwörter: elektrische Maschinen; Verlustbestimmung; Ummagnetisierungsverluste; magnetischer Kreis; Einzelzahn

\section{Realistic determination of the iron losses of an electric machine with single teeth.}

High technical and economic demands are present for electrical machines in electrified automotive drives. On the one hand, methods that allow the production of large quantities while maintaining low costs are required. On the other hand, electrical drive trains with high efficiency and high power density are needed to provide for a long electrical range. Prerequisite for the development of electrical machines with the above-mentioned characteristics are detailed loss models. Especially the modelling of iron losses in the stator and rotor of the machine is challenging. In addition to the choice of materials, the manufacturing processes have a significant influence on the loss characteristics of the machine. This publication presents a new approach for the determination of iron losses of a single stator tooth under consideration of realistic flux conditions. With the presented method, manufacturing influences on the iron losses can be investigated while maintaining the application-specific geometry and the magnetic utilization of the tooth. At first, the magnetic conditions of an electric machine with single tooth winding are identified. Subsequently, the single-tooth test bench is introduced and the transfer of relevant magnetic quantities of the machine to the test bench is presented. The determination of tooth losses based on measured loss data is shown. Finally, we report a comparison of simulation and measurement results. The presented method offers the possibility to adapt iron loss models to additional manufacturing-related losses and to reduce uncertainties in iron loss modelling in general.

Keywords: electrical machines; loss measurement; core loss; magnetic circuit; single tooth

Eingegangen am 9. April 2020, angenommen am 23. April 2020, online publiziert am 12. Mai 2020

(c) The Author(s) 2020

\section{Einleitung und Problemstellung}

Um große Reichweiten zu ermöglichen, muss sich der elektrische Antriebsstrang eines Batterie- oder Hybridelektrischen Fahrzeugs durch hohe Effizienz und niedriges Gewicht auszeichnen. Die Steigerung der Leistungsdichte kann durch eine höhere elektromagnetische Ausnutzung der Maschine oder eine Erhöhung der Drehzahl erreicht werden. Die Auslegung des Kühlsystems muss dabei die steigenden Maschinenverluste berücksichtigen. Dies Bedarf den Einsatz von Verlustmodellen, mit welchen die Maschinenverluste anwendungsnah beschrieben werden könne. Neben den Stromwärmeverlusten haben in elektrischen Maschinen die Ummagnetisierungsverluste, im Folgenden abkürzend Eisenverluste genannt, einen großen
Anteil an den gesamten Maschinenverlusten und müssen daher detailliert modelliert werden können.

Gleichzeitig müssen neben der Steigerung der Leistungsdichte Produktionsmethoden gefunden werden, welche die Fertigung hoher Stückzahlen bei Einhaltung niedriger Produktionskosten ermög-

Rollbühler, Christoph, Elektrotechnisches Institut, Karlsruhe Institut für Technologie, Karlsruhe, Deutschland (E-Mail: c.rollbuehler@kit.edu); Breining, Patrick,

Elektrotechnisches Institut, Karlsruhe Institut für Technologie, Karlsruhe, Deutschland (E-Mail: patrick.breining@kit.edu); Kolb, Johannes, Schaeffler Technologies AG \& Co. KG (SHARE at KIT), Karlsruhe, Deutschland; Doppelbauer, Martin, Elektrotechnisches Institut, Karlsruhe Institut für Technologie, Karlsruhe, Deutschland 
lichen. Die Stator- und Rotorpakete elektrischer Maschinen werden üblicherweise aus gestapelten Elektroblechen hergestellt. Diese Silizium-Eisen Legierungen ermöglichen eine gute Führung des magnetischen Flusses bei niedrigen Eisenverlusten. Untersuchungen verschiedener Trennverfahren für Elektrobleche haben gezeigt, dass je nach Verfahren eine deutliche Verschlechterung der magnetischen Eigenschaften eintritt [1-3].

Für die Bestimmung der magnetischen Eigenschaften von weichmagnetischen Werkstoffen gibt es standardisierte Prüfverfahren. Dazu zählen die Vermessung von schmalen Blechstreifen mit dem Epstein Rahmen [4] oder von quadratischen Blechscheiben mit dem Einzeltafelmessgerät [5]. Diese Messverfahren liefern zunächst nur die reinen Materialeigenschaften der untersuchten Elektrobleche, ein Einfluss des Fertigungsverfahrens kann nur mit Hilfe weiterführender Messungen untersucht werden. In [6] wurden die Blechstreifen für die Vermessung am Epstein Rahmen weiter unterteilt um in der Probe einen größeren Anteil an Schnittkanten zu erhalten. Wird der Schnittkantenanteil der Probe variiert, können Aussagen zum Einfluss des Trennverfahrens auf die Eisenverluste abgeleitet werden.

Eine weitere standardisierte Methode zur Charakterisierung der Trennprozesse ist die Untersuchung von Ringkernen [7]. Hier wird das Elektroblech zunächst in Ringe geschnitten und anschließend zu einem Kern gestapelt. Durch den Vergleich unterschiedlicher Ringbreiten mit variierendem Schnittkantenanteil kann der Einfluss des Trennverfahrens ermittelt werden [8]. Des Weiteren können mit Ringproben die magnetischen Eigenschaften von Pulverwerkstoffen ermittelt werden [9]. Neben dem Trennverfahren kann auch das Paketieren von Elektroblechen zu gesteigerten Eisenverlusten führen. In [10] wurden Ringproben genutzt, um den Einfluss verschiedener Fügeprozesse auf die Wirbelstromverluste im Blechpaket zu untersuchen.

Die Ergebnisse der genannten Untersuchungen zeigen, dass Fertigungsprozesse einen signifikanten Einfluss auf die Eisenverluste in elektrischen Maschinen haben. Im Unterschied zu den tatsächlich in elektrischen Maschinen eingesetzten Blechschnitten und Fertigungsschritten, können bei den standardisierten Aufbauten jedoch nur einfache rechteck- oder ringförmige Geometrien untersucht werden. Außerdem wird für die Messungen eine homogene Verteilung der magnetischen Flussdichte, sowie eine rein wechselnde Magnetisierung in der Probe eingestellt. Der Geometrieaspekt kann teilweise gelöst werden, indem Messungen an fertigen Statorpaketen durchgeführt werden. Dazu wird das Statorjoch als Ringkern angenommen und entsprechend magnetisch charakterisiert [11, 12]. Da die räumliche und zeitliche Verteilung der Flussdichte aber weiterhin nicht der in der realen Anwendung entspricht, werden weiterführende Ansätze benötigt, um die Eisenverluste in der Maschine exakt bestimmen zu können.

In den Augen der Autoren stehen dafür zwei Ansätze zur Verfügung. Zum einen kann die elektrische Maschine an einem Maschinenprüfstand betrieben werden und so die Gesamtverluste über das Betriebskennfeld ermittelt werden. Dies ist allerdings mit Unsicherheiten verbunden, da die Eisenverluste zunächst von anderen frequenz- und lastabhängigen Verlusten getrennt werden müssen. Dies betrifft insbesondere die korrekte Abbildung der mechanischen Verluste und der Stromverdrängungsverluste in der Statorwicklung der Maschine. Der zweite Ansatz nutzt die Tatsache, dass ein einzelner Statorzahn die kleinste sich wiederholende Einheit im Statorpaket einer elektrischen Maschine darstellt. Kann ein Statorzahn in allen relevanten Betriebspunkten der Maschine magnetisch charakterisiert werden, so lassen sich die Eisenverluste des Stators gezielt ermitteln. Da ein Einzelzahn zusätzlich alle verlustrelevanten fertigungsbedingten Effekte aufweist, kann auf diese Weise ebenfalls eine Aussage zu den zusätzlichen Verlusten getroffen werden.
Dazu wurde am Elektrotechnischen Institut des Karlsruher Instituts für Technologie (KIT) ein Einzelzahn-Prüfstand entwickelt [13]. Mit diesem können Statorzähne in ihrem endgültigen Zustand magnetisch charakterisiert werden. Da die Proben den spezifischen Einfluss des verwendeten Trenn- und Fügeverfahrens aufweisen, können die Eisenverluste der Einzelzähne realitätsnah bestimmt werden. In bisherigen Untersuchungen wurden Arbeitspunkte angelehnt an den Leerlauf der Maschine betrachtet. Diese zeichnen sich durch einen oftmals sinusförmigen Verlauf der magnetischen Flüsse aus. So konnten verschiedene Herstellungsverfahren unter Leerlaufbedingungen verglichen werden.

In diesem Beitrag wird die Erweiterung des Messverfahrens auf alle relevanten Arbeitspunkte der Maschine vorgestellt. Im Fokus steht dabei die Identifizierung der in der Maschine auftretenden magnetischen Flüsse und deren Übertragung auf den Prüfstand. Anhand einer exemplarischen Maschine werden die relevanten Größen zur Beschreibung der magnetischen Flüsse eines Einzelzahns im Maschinenbetrieb definiert und abgeleitet. Die Berücksichtigung der Oberschwingungen ist insbesondere im Feldschwächebetrieb zwingend erforderlich. Anschließend wird der Aufbau des Prüfstands erläutert, wobei der elektromagnetische Kreis sowie das Mess- und Regelsystem im Vordergrund stehen. Zur weiteren Auswertung der Messdaten wird die Implementierung eines Trennverfahrens für Jochund Zahnverluste vorgestellt. Im Ergebniskapitel werden Messungen verschiedener Betriebspunkte gezeigt und mit den zuvor aus der Maschinensimulation abgeleiteten Flussverläufen verglichen. Außerdem wird die Aufteilung der Verluste in Joch- und Zahnverluste vorgenommen.

Das Verfahren bietet somit die Möglichkeit, Statorzähne unter Berücksichtigung des Herstellungsverfahrens und des Betriebspunktes magnetisch zu charakterisieren und zu bewerten. Dies ermöglicht zum einen die Quantifizierung des Fertigungseinflusses unter realen Bedingungen. Zum anderen können mit den erlangten Messdaten bestehende Eisenverlustmodelle validiert und erweitert werden.

\section{Methodik und Diskussion}

\subsection{Flussverhältnisse in elektrischen Maschinen}

Anhand einer permanentmagneterregten Synchronmaschine aus dem Antriebsstrang eines Elektrofahrzeuges mit einer Nennleistung von $11 \mathrm{~kW}$ werden im Folgenden grundsätzliche Zusammenhänge und Definitionen bezüglich der Eisenverluste in einem Einzelzahn des Stators veranschaulicht. Der Stator der Maschine besteht aus 24 Einzelzähnen, es kommt eine konzentrierte Wicklung zum Einsatz. Die mittlere Breite eines Statorzahns beträgt am Zahnfuß 15,1 mm und am Zahnrücken $10 \mathrm{~mm}$. Die Maschine besitzt 10 Polpaare und eine aktive Eisenlänge von 45,6 mm, die Maximaldrehzahl liegt bei $7000 \mathrm{~min}^{-1}$.

Ausgehend vom Einzelzahn, als kleinste sich wiederholende Komponente des Stators, können wie in Abb. 1 dargestellt die Flüsse $\phi_{\mathrm{A}}$ und $\phi_{\mathrm{B}}$ am Zahnrücken, wie auch der Fluss $\phi_{\text {Tf }}$ am Zahnfuß definiert werden. Die Flüsse durch die drei rot dargestellten Schnittflächen genügen um die Flussverhältnisse des Zahns vollständig zu charakterisieren. Des Weiteren wurde in [14] eine Berechnungsvorschrift für den Phasenversatz zwischen den Flüssen auf A- und B-Seite für den Leerlauffall definiert. Der hochgestellte Index vor dem Formelzeichen gibt dabei die Ordnungszahl der Harmonischen an. Für die Grundwelle ergibt sich:

$$
{ }^{1} \gamma=180^{\circ} \cdot\left(1-\frac{1}{q \cdot m}\right)={ }^{1} \gamma_{A}-{ }^{1} \gamma_{B}
$$

Hierbei ist $m$ die Phasenzahl und $q$ die Lochzahl der Maschine, im Falle der betrachteten Maschine entspricht $m=3$ und $q=0,4$. 


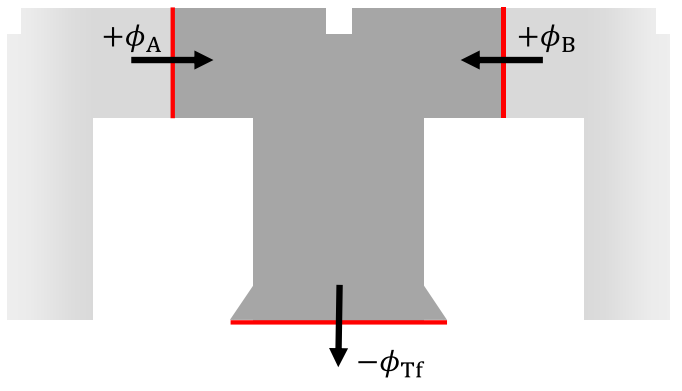

Abb. 1. Schematische Darstellung eines Einzelzahns

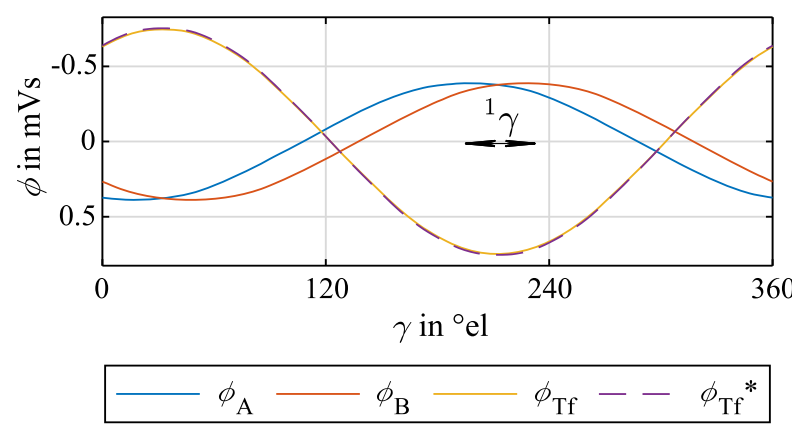

Abb. 2. Zeitverlauf der Flüsse des Einzelzahns im Leerlauf, Simulation. $\phi_{\mathrm{A}}$ : Jochfluss, A-seitig; $\phi_{\mathrm{B}}$ : Jochfluss, B-seitig; $\phi_{\mathrm{Tf}}$ : Zahnfußfluss; $\phi_{\mathrm{Tf}}^{*}$ Zahnfußfluss berechnet unter Annahme eines linearen magnetischen Kreises

Es ergibt sich also ein Leerlaufphasenversatz von ${ }^{1} \gamma=30^{\circ} \mathrm{el}$, wie in Abb. 2 dargestellt. Außerdem ist in Abb. 2 der Zahnfußfluss in lila bzw. gelb dargestellt. Dieser entspricht im linearen Fall der Summe der Flüsse auf A- und B-Seite: $\phi_{T f}^{*}=-\left(\phi_{A}+\phi_{B}\right)$. Wird das Material jedoch im nichtlinearen Bereich betrieben, kann der Verlauf davon abweichen (gelbe Linie). Die Verläufe wurden per Finite-Elemente Berechnung ermittelt.

Im Vergleich zum Leerlauffall verändern sich die Zeitverläufe der Flüsse unter Last jedoch stark, wie in Abb. 3 und 4 zu sehen. Abb. 3 zeigt die Verläufe im Nennpunkt der Maschine und Abb. 4 zeigt die Verläufe bei maximalem Drehmoment und maximaler Geschwindigkeit. Im Vergleich zum Leerlauf ist im Nennpunkt eine merklich höhere Flussamplitude vorherrschend was zu einem größeren Einfluss der Nichtlinearitäten des Eisens führt. Dadurch entspricht wie oben angedeutet das aus $\phi_{\mathrm{A}}$ und $\phi_{\mathrm{B}}$ berechnete $\phi_{\mathrm{Tf}}^{*}$ nicht mehr dem wirklichen Verlauf $\phi_{\mathrm{Tf}}$. Die Differenz kann als Streufluss definiert werden:

$$
\phi_{\sigma, \mathrm{Tf}}=\phi_{T f}^{*}-\phi_{T f}
$$

Bei Betrieb in der Feldschwächung wird der Fluss im Zahn durch einen negativen d-Strom geschwächt, die Amplitude nimmt ab und zusätzliche Harmonische entstehen, vergleiche Abb. 4. In beiden Lastfällen ändert sich auch die Phasenlage zwischen $\phi_{\mathrm{A}}$ und $\phi_{\mathrm{B}}$. So ist sie im Nennpunkt nahezu Null, bei Maximaldrehzahl dreht sich das Vorzeichen der Phase und Fluss $\phi_{A}$ eilt $\phi_{B}$ vor. Zusammenfassend sind also folgende Größen notwendig um den Flussverlauf innerhalb der Einzelzahngeometrie zu definieren:

- Flüsse $\phi_{A}, \phi_{B}, \phi_{\text {Tf }}$ im Zahn, sowie die entsprechenden Flussdichten $B_{\mathrm{A}}, B_{\mathrm{B}}, B_{\mathrm{Tf}}$

- Phasenversatz der Grundwelle zwischen $\phi_{A}$ und $\phi_{B}:{ }^{1} \gamma$

- Streufluss $\phi_{\sigma, \text { Tf }}$ Zwischen Zahnrücken und Zahnfuß

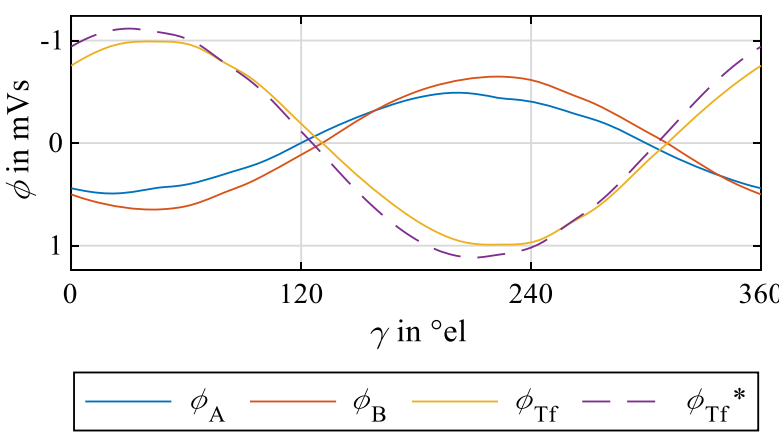

Abb. 3. Zeitverlauf der Flüsse des Einzelzahns im Nennpunkt, Simulation. $\phi_{\mathrm{A}}$ : Jochfluss, A-seitig; $\phi_{\mathrm{B}}$ : Jochfluss, B-seitig; $\phi_{\mathrm{Tf}}$ : Zahnfußfluss; $\phi_{\mathrm{Tf}}^{*}:$ Zahnfußfluss berechnet unter Annahme eines linearen magnetischen Kreises

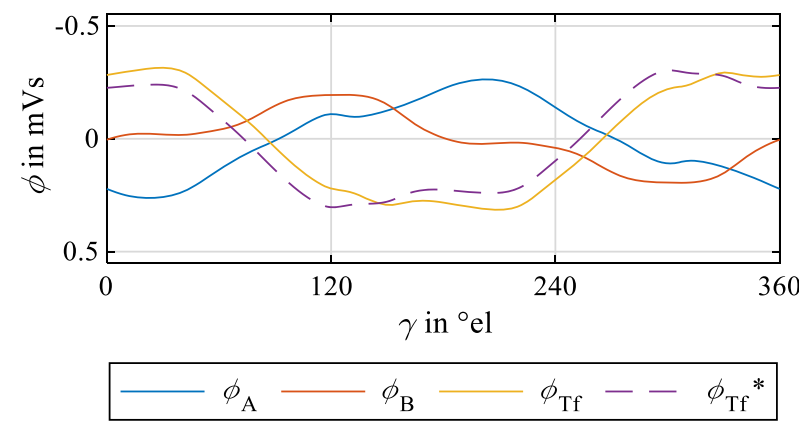

Abb. 4. Zeitverlauf der Flüsse des Einzelzahns bei maximalem Drehmoment und maximaler Geschwindigkeit, Simulation. $\phi_{\mathrm{A}}$ : Jochfluss, A-seitig; $\phi_{\mathrm{B}}$ : Jochfluss, B-seitig; $\phi_{\mathrm{Tf}}$ : Zahnfußfluss; $\phi_{\mathrm{Tf}}^{*}$ : Zahnfußfluss berechnet unter Annahme eines linearen magnetischen Kreises

- Oberwellengehalt des Flusses: Berechnung der Total Harmonic Distortion (THD)

Ausgehend von den unterschiedlichen Flussverläufen je Betriebspunkt sind die Anforderungen an einen Prüfstand mit dem Ziel der Nachbildung dieser Flussverläufe hoch. Variable Amplituden, Frequenzen und Phasendifferenzen sind ebenso erforderlich wie die Möglichkeit verschiedene Harmonische einzuprägen. Die Grundlage für die umfängliche magnetische Charakterisierung von Einzelzähnen bildet der in [13] vorgestellte Einzelzahn-Prüfstand. Im folgenden Abschnitt werden grundsätzliche Funktionsweise des Prüfstands sowie notwendige Erweiterungen vorgestellt.

\subsection{Prüfstand für Einzelzähne}

Der Einzelzahnmessplatz besteht aus zwei Jochhälften, welche den magnetischen Kreis über den zu untersuchenden Zahn schließen. Die Jochhälften werden individuell an die Zahngeometrie angepasst. Über einen pneumatischen Zylinder kann die Probe mit definiertem Druck zwischen die Jochhälften gespannt werden. Die magnetische Erregung wird über die beiden Primärwicklungen an den Jochseiten links und rechts des Zahns eingestellt. Mittels den als Messspulen dienenden Sekundärspulen am Zahnjoch sowie am Zahnfuß können die entsprechenden magnetischen Flüsse ermittelt werden. Der schematische Aufbau des Messsystems ist in Abb. 5 dargestellt.

\subsubsection{Elektromagnetischer Kreis}

Die magnetischen Flüsse $\phi_{A}, \phi_{B}$ und $\phi_{T f}$ im Einzelzahn können unter Zuhilfenahme der induzierten Spannungen $u_{2, A}, u_{2, B}$ und $u_{2, T f}$ 


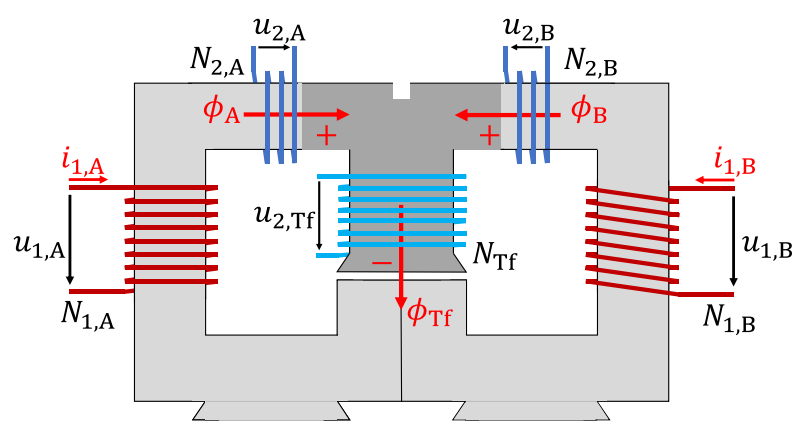

Abb. 5. Schematische Darstellung des Prüfstands für Einzelzähne

in den jeweiligen Messwicklungen mit den Windungszahlen $N_{2, A}$ $N_{2, B}$ und $N_{\text {Tf }}$ ermittelt werden. Die Magnetisierung des Aufbaus wird über die beiden Primärwicklungen mit den Windungszahlen $N_{1, A}$ und $N_{1, B}$ und die eingeprägten Primärströme $i_{1, A}$ und $i_{1, B}$ eingestellt. Zusätzlich stehen als Messgrößen die Spannungen der Primärwicklungen $u_{1, A}$ und $u_{1, B}$ zur Verfügung. Für eine kompaktere Beschreibung der beiden Jochseiten wird der Bezeichner $x \in\{A, B\}$ eingeführt. Die gesuchten Flüsse ergeben sich somit über folgenden Zusammenhang:

$$
u_{2, x}(t)=N_{2, x} \cdot \frac{\mathrm{d} \phi_{x}(\mathrm{t})}{\mathrm{d} t}
$$

Neben der Flussamplitude ist auch die Phasenlage der beiden Flusskomponenten $\phi_{A}$ und $\phi_{B}$ für die weitere Betrachtung relevant. Die Phasenlage ist ein Indikator für die Ausbildung rotierender Flussanteile im Zahnrücken des Einzelzahns. Die Referenzphase ist dabei die Phasenlage des Grundwellenflusses der Jochseite A. Zusätzlich kann mit der Spule am Zahnfuß eine Aussage über Zahnstreuflüsse getroffen werden. Dazu werden die magnetischen Flüsse am Zahnjoch und am Zahnfuß in Relation gesetzt, entsprechend Gl. (2) kann der Streuanteil bestimmt werden.

Über die Leistungsgleichung des Gesamtsystems können die einzelnen Anteile ermittelt werden. Die ohmschen Verluste in den Primärwicklungen sind dabei abhängig von den jeweiligen Wicklungswiderständen $R_{1, \mathrm{~A}}$ und $R_{1, \mathrm{~B}}$ :

$$
\underbrace{\sum_{x \in[\mathrm{A}, \mathrm{B}]} u_{1, x} \cdot i_{1, x}}_{\text {Systemleistung }}=\underbrace{\sum_{x \in[\mathrm{A}, \mathrm{B}]} R_{1, x} \cdot i_{1, x}^{2}(\mathrm{t})}_{\text {Ohmsche Verlustleistung }}+\underbrace{\sum_{x \in[\mathrm{A}, \mathrm{B}]} \frac{N_{1, x}}{N_{2, x}} u_{2, x} \cdot i_{1, x}}_{\text {Magnetisierungsleistung }}
$$

Der Mittelwert der Magnetisierungsleistung über eine Signalperiode $T$ liefert die Eisenverluste des Systems. Durch die Verwendung der Sekundärspannungen können die ohmschen Verlustanteile von der Magnetisierungsleistung getrennt werden. Die Eisenverluste des Aufbaus sind somit:

$$
P_{\mathrm{fe}}=\sum_{x \in[\mathrm{A}, \mathrm{B}]} \frac{1}{T} \int_{t_{0}}^{t_{0}+T} \frac{N_{1, x}}{N_{2, x}} u_{2, x}(\mathrm{t}) \cdot i_{1, x}(\mathrm{t}) \mathrm{dt}
$$

\subsubsection{Regelung}

Für eine exakte Nachbildung der magnetischen Zustände im Einzelzahn müssen definierte Flussverläufe an den Übergängen von Messjoch zu Zahnrücken eingestellt werden. Da die magnetischen Flüsse in Abhängigkeit des Arbeitspunkts der Maschine signifikante Oberwellenanteile aufweisen können, ist am Einzelzahnmessplatz die Einstellung beliebiger Flussharmonischer möglich. Die Ergebnisse der simulativen Maschinenuntersuchung haben gezeigt, dass für eine detaillierte Beschreibung der Flusscharakteristik Harmonische bis zur siebten Ordnung benötigt werden. Zusätzlich muss für die Erzeugung von Drehfeldern im Zahnrücken eine unabhängige Regelung der beiden Seiten A und B durchgeführt werden. Die Umsetzung am Prüfstand erfolgt mit einem iterativen Regelalgorithmus auf Basis der Repetitive Control Strategy [13]. Die folgende Definitionsgleichung wird als Grundlage für die zu regelnden Flusskurvenformen verwendet:

$$
\begin{gathered}
\phi_{A}(t)={ }^{1} \hat{\phi}_{A}(t) \cdot \sin (\omega t)+{ }^{3} \hat{\phi}_{A}(t) \cdot \sin \left(3 \omega t+{ }^{3} \gamma_{A}\right) \\
+{ }^{5} \hat{\phi}_{A}(t) \cdot \sin \left(5 \omega t+{ }^{5} \gamma_{A}\right) \\
+{ }^{7} \hat{\phi}_{A}(t) \cdot \sin \left(7 \omega t+{ }^{7} \gamma_{A}\right) \\
\phi_{B}(t)={ }^{1} \hat{\phi}_{B}(t) \cdot \sin \left(\omega t+{ }^{1} \gamma\right)+{ }^{3} \hat{\phi}_{B}(t) \cdot \sin \left(3 \omega t+{ }^{1} \gamma+{ }^{3} \gamma_{B}\right) \\
+{ }^{5} \hat{\phi}_{B}(t) \cdot \sin \left(5 \omega t+{ }^{1} \gamma+{ }^{5} \gamma_{B}\right) \\
+{ }^{7} \hat{\phi}_{B}(t) \cdot \sin \left(7 \omega t+{ }^{1} \gamma+{ }^{7} \gamma_{B}\right)
\end{gathered}
$$

Dabei gibt der hoch vorangestellte Index vor Flussamplitude $\hat{\phi}_{x}$ und Phasenlage $\gamma_{x}$ die Ordnungszahl der jeweiligen Harmonischen an, $\omega$ bezeichnet die elektrische Winkelgeschwindigkeit der Grundwelle. Im stationären Betrieb sind die Flussverläufe der Maschine periodisch. Diese Periodizität der Sollwerte ist Voraussetzung für die Anwendung eines Regelverfahrens mit der oben eingeführten repetitiven Regelstrategie. Der Ablauf des Regelverfahrens beinhaltet:

1. Bestimmung der Abweichung zwischen Soll- und Istwerten innerhalb einer Signalperiode an N Stützstellen

2. Berechnung des Korrekturwertes und Anpassung der Stellgröße an den $N$ Stützstellen

3. Erreichen von Stationarität nach einer Stellgrößenänderung

4. Überwachung des Regelfehlers der aktuellen Iteration und der Abbruchbedingung

Der Korrekturwert kann mit der Newton-Methode ermittelt werden. Es ist zu beachten, dass die Fehlerberechnung im stationären Zustand durchgeführt wird. Daher können die ersten Perioden nach einer Stellgrößenänderung nicht zur Fehlerbestimmung verwendet werden.

Da die beiden Primärwicklungen A und B über den Prüfling magnetisch gekoppelt sind, wird das beschriebene Verfahren abwechselnd für die Seiten A und B durchgeführt, bis die Regelabweichung für beide Wicklungen minimal ist. Dazu wird die Steigung der Regelabweichung ausgewertet und der Regelvorgang bei Erreichen des Minimums abgebrochen.

Die Regelstrategie ist auf einem Field Programmable Gate Array (FPGA) vom Typ Cyclone IV am Einzelzahn-Prüfstand implementiert. Dies ermöglicht die Verwendung von $N=1000$ Interpolationspunkten pro Signalperiode und damit eine hohe zeitliche Auflösung der Messgrößen bis zur Abtastrate der Analog-Digital-Wandler von 1,2 MSps. Insbesondere bei hohen Grundfrequenzen und zusätzlichen Oberwellen stellt dies hohe Anforderungen an die Signalverarbeitung und Steuerung [13]

Die Kommunikation und die Steuerung des Prüfstands sind mit einem eigens entwickelten digitalen Signalverarbeitungssystem realisiert [15]. Linearverstärker mit einer Bandbreite größer $100 \mathrm{kHz}$ und Signalanstiegszeiten von bis zu $100 \mathrm{~V} / \mu$ s bilden den Leistungsteil. Über eine Parallelschaltung von bis zu vier Linearverstärkermodulen pro Seite kann ein Ausgangsstrom von bis zu 200 A bei einer Ausgangsspannung von maximal $60 \mathrm{~V}$ erreicht werden [13]. 


\subsection{Separation der Zahnverluste}

Die Eisenverluste des Aufbaus können wie beschrieben mit GI. (5) ermittelt werden. Darin sind neben den gesuchten Eisenverlusten im Zahn auch die Eisenverluste der Prüfjoche enthalten. Der Einfluss des Prüfjochs kann minimiert werden, indem Elektrobleche mit hoher Sättigungspolarisation und niedrigen Eisenverlusten eingesetzt werden.

Zusätzlich besteht die Möglichkeit der Separation der Grundwelleneisenverluste im Einzelzahn. Die Grundidee des Ansatzes ist die Einführung eines digitalen Abbilds des Messaufbaus in einer geeigneten Simulationsumgebung. Kann für alle Betriebspunkte sichergestellt werden, dass die gemessenen Verluste im Simulationsmodell abgebildet werden können, ist eine Trennung der Zahn- von den Jochverlusten möglich. Da sich der Betriebsbereich der Maschine über verschiedene Frequenzen, Amplituden der Flussdichten und Phasenlagen erstreckt, muss das Modell für diese Betriebspunkte valide sein.

Für das Verfahren wird ein Referenzzahn benötigt. Dieser sollte aus dem gleichen Material und mit den gleichen Fertigungsmethoden wie die Prüfjoche hergestellt werden. Im Simulationsmodell werden die Eisenverluste mit Hilfe des Bertotti Modells ermittelt [16]. Im Frequenzbereich lautet die Modellgleichung für die spezifischen Eisenverluste $P_{\mathrm{fe}, \mathrm{s}}$ in Abhängigkeit von Frequenz $f$ und Flussdichte $B$ :

$$
P_{\mathrm{fe}, \mathrm{s}}=k_{\text {hys }} \cdot B^{2} f+k_{\mathrm{cls}} \cdot B^{2} f^{2}+k_{\mathrm{exc}} \cdot B^{1.5} f^{1.5}
$$

Die gesamten Eisenverluste werden unterteilt in Hystereseverluste $\left(k_{\text {hys }}\right)$, klassische Wirbelstromverluste $\left(k_{\mathrm{cls}}\right)$ und Zusatzverluste $\left(k_{\text {exc }}\right)$. Der Ablauf des Verfahrens lässt sich folgendermaßen zusammenfassen:

1. Bestimmung der Grundwelleneisenverluste $P_{\text {mess }}$ des Gesamtsystems mit dem Referenzzahn. Berücksichtigt werden Frequenzen und Flussdichten aus dem Betriebsbereich der Maschine, sowie verschiedene Phasenlagen $\gamma$.

2. Modellierung des Gesamtaufbaus in der Finite-Elemente Software Altair Flux. Basierend auf den CAD Zeichnungen, wird ein 2DModell des Aufbaus abgeleitet. Die Positionen von Primär- und Sekundärwicklungen werden berücksichtigt.

3. Die Arbeitspunkte aus Schritt 1 werden nun im Simulationsmodell untersucht. Dabei muss sichergestellt werden, dass der zeitliche Verlauf der magnetischen Flüsse exakt nachgebildet wird.

4. Bestimmung der Modellverluste $P_{\text {sim }}$ mit den Modellparametern der Verlustgleichung (8). Der Parametervektor $\vec{k}=\left(k_{\text {hys }}, k_{\mathrm{cls}}, k_{\mathrm{exc}}\right)$ ist die gesuchte Lösung des Optimierungsproblems

$$
\min _{\vec{k}} \operatorname{abs}\left(P_{\text {sim }}(B, f, \gamma, \vec{k})-P_{\text {mess }}(B, f, \gamma)\right) .
$$

Mit dem ermittelten optimalen Parametervektor $\vec{k}$ können nun die Eisenverluste der Prüfjoche $P_{\text {Joch }}$ und des Zahns $P_{\text {Zahn }}$ berechnet werden. Zusätzlich kann der Jochverlustfaktor $k_{\text {Joch }}$ angegeben werden. Dieser setzt die Jochverluste in Relation zu den Zahnverlusten:

$$
k_{\text {Joch }}=\frac{P_{\text {Joch }}(B, f, \gamma, \vec{k})}{P_{\text {Zahn }}(B, f, \gamma, \vec{k})}
$$

\subsection{Messergebnisse}

\subsubsection{Einprägung von Harmonischen}

Die eingangs hergeleiteten und simulierten Flussverhältnisse im Zahn lassen sich per Fourier Analyse in die einzelnen auftretenden Oberschwingungen zerlegen und gemäß Gl. (6) und (7) am Prüfstand einregeln. Die gemessenen Flussverläufe sind in Abb. 6

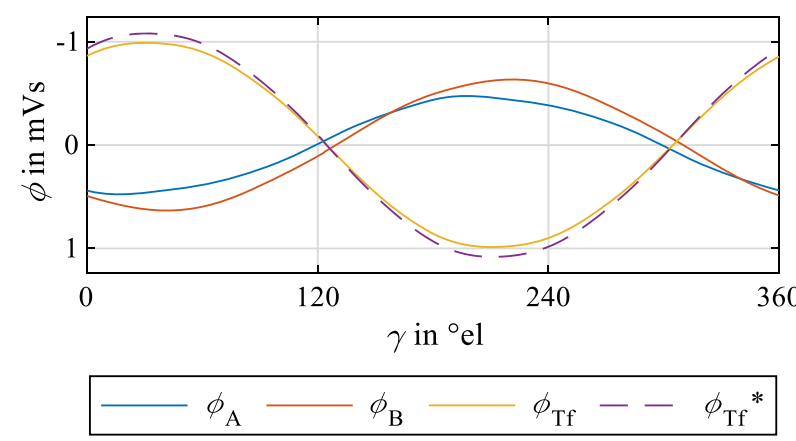

Abb. 6. Zeitverlauf der Flüsse des Einzelzahns im Nennpunkt, Messung. $\phi_{\mathrm{A}}$ : Jochfluss, A-seitig; $\phi_{\mathrm{B}}$ : Jochfluss, B-seitig; $\phi_{\mathrm{Tf}}$ : Zahnfußfluss; $\phi_{\mathrm{Tf}}^{*}$ : Zahnfußfluss berechnet unter Annahme eines linearen magnetischen Kreises

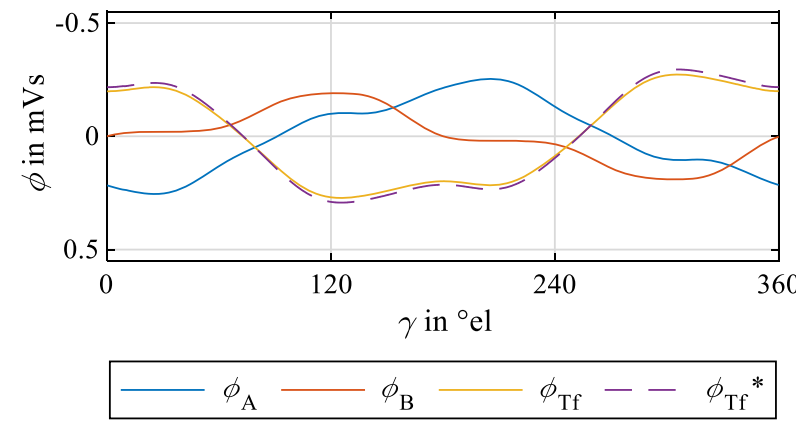

Abb. 7. Zeitverlauf der Flüsse des Einzelzahns bei maximalem Drehmoment und maximaler Geschwindigkeit, Messung. $\phi_{\mathrm{A}}$ : Jochfluss, $\mathrm{A}-$ seitig; $\phi_{\mathrm{B}}$ : Jochfluss, B-seitig; $\phi_{\mathrm{Tf}}$ : Zahnfußfluss; $\phi_{\mathrm{Tf}}^{*}:$ Zahnfußfluss berechnet unter Annahme eines linearen magnetischen Kreises

und 7 dargestellt und weisen nur geringfügige Abweichungen im Vergleich zur Simulation auf. Auch beim Punkt maximalem Drehmoments und maximaler Geschwindigkeit (Abb. 7), welcher einen hohen Oberwellengehalt aufweist, ermöglichen die ersten sieben Harmonischen eine ausreichend gute Signalrekonstruktion. Die Abweichungen der jeweiligen Amplituden der Harmonischen zwischen Sollwert aus der Simulation und gemessenem Zeitverlauf am Prüfstand sind kleiner als 3,5\%.

\subsubsection{Bestimmung der Zahnverluste}

Generell gilt es die Verluste in den Prüfjochen zu minimieren. Daher bieten sich Fertigungsverfahren an, die wenig Einfluss auf die Eisenverluste des Referenzmaterials haben. Als Verfahren mit geringem Einfluss auf die magnetischen Eigenschaften gelten Drahterosion und Wasserstrahlschneiden [2]. Im Vergleich zu gängigen Trennverfahren wie Stanzen oder Laserschneiden können so die Verluste im Prüfjoch reduziert werden, die Verfahren sind dabei allerdings kostenintensiver. Durch die Verwendung des Separationsansatzes können die Zahnverluste von den Jochverlusten abgetrennt werden. Der Einfluss der Prüfjoche kann so zusätzlich reduziert werden.

Aufgrund von Kosten und Verfügbarkeit wurden die Prüfjoche und der Referenzzahn für die betrachtete Geometrie mittels Laserschneiden angefertigt. Die Blechschnitte wurden anschließend unter Verwendung von Backlack paketiert. Die Prüfjoche des Aufbaus sind ebenso wie der Referenzzahn aus NO20 gefertigt. Durch die geringe Dicke der Bleche von 0,2 mm können die auftretenden Wirbelstrom- 


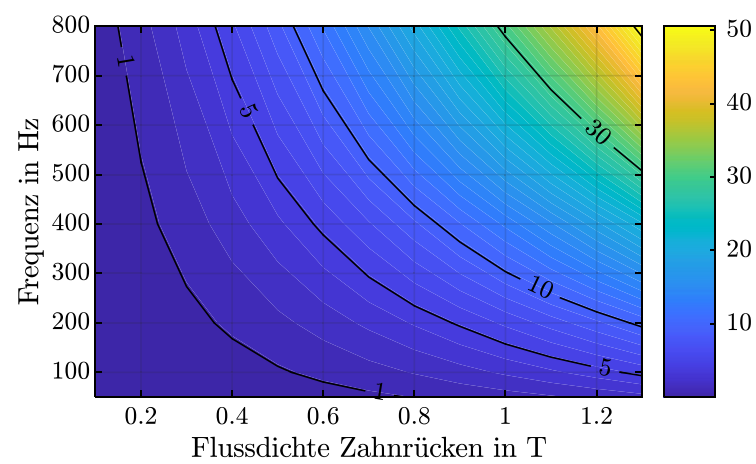

Abb. 8. Gesamteisenverluste mit Referenzzahn bei $0^{\circ}$ Phasenlage in $W / k g$

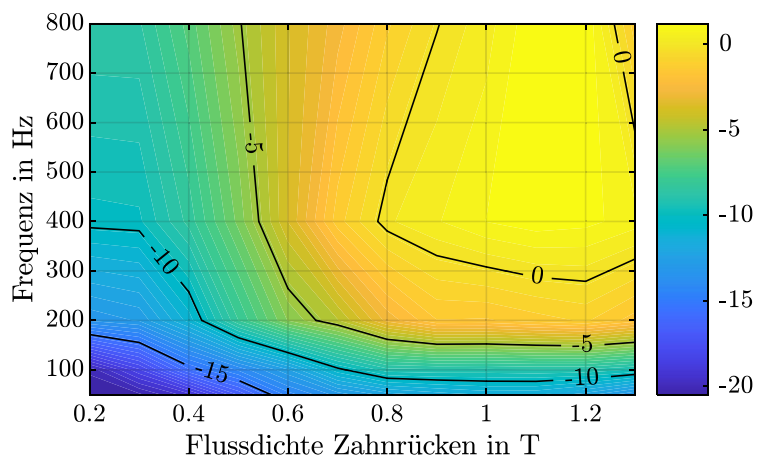

Abb. 9. Relative Modellabweichung bei $0^{\circ}$ Phasenlage in $\%$

verluste im Blech gemindert werden. So können auch Arbeitspunkte mit hohen Frequenzanteilen untersucht werden, ohne übermäßige Verluste in den Prüfjochen zu erzeugen.

Für die Separation der Zahnverluste werden die Verlustkennfelder des Referenzmaterials benötigt. In Abb. 8 sind die spezifischen Eisenverluste des Gesamtaufbaus dargestellt. Es wurden dabei Frequenzen bis $800 \mathrm{~Hz}$ berücksichtig, die maximale Flussdichte im Zahnrücken beträgt 1,3 T. Anschließend können die Verlustkoeffizienten mit Gl. (9) ermittelt werden. Die Differenz aus gemessenen Gesamtverlusten und Modellverlusten ist ein Indikator für die Genauigkeit des Ansatzes. In Abb. 9 ist dazu der relative Modellfehler der Gesamtverluste gezeigt. Insbesondere der Bereich hoher Frequenzen und Flussdichten kann mit dem gewählten Verlustmodell gut beschrieben werden. Der absolute Modellfehler im Kennfeld ist stets kleiner als 0,5 W/kg.

Mit den Verlustkoeffizienten können die Gesamtverluste in Zahnund Jochverluste unterteilt werden. In Abb. 10 ist der Jochverlustfaktor für $0^{\circ}$ Phasenlage gemäß Gl. (10) dargestellt. Die Eisenverluste im Joch sind etwa um den Faktor 2,15 größer als die Zahnverluste. Mit den ermittelten Daten für die Jochverluste können nun weitere Einzelzähne aus anderen Materialien oder mit anderen Fertigungsverfahren untersucht werden. Die gemessenen Gesamtverluste werden um den entsprechenden Modellwert der Jochverluste korrigiert. In Abb. 11 sind die spezifischen Zahnverluste für Referenzzahn und exemplarisch für einen Probenzahn (Zahn 1) angegeben. Zusätzlich sind die korrespondierenden Jochverluste gezeigt. Zahn 1 ist in diesem Fall aus dem Material M250-35A hergestellt, die Bleche wurden gestanzt und verklebt. Werden weitere Fertigungs- oder Fügeprozesse mit in die Untersuchung aufgenommen, können die Einflüsse auf die Eisenverluste anwendungsspezifisch ermittelt und unter-

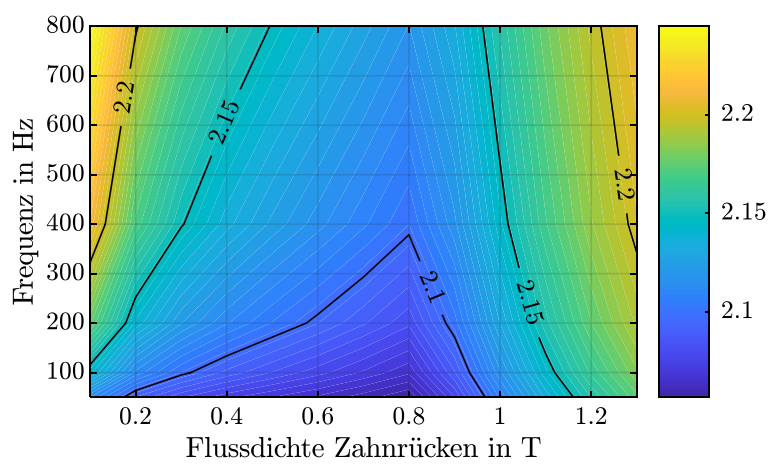

Abb. 10. Jochverlustfaktor bei $0^{\circ}$ Phasenlage

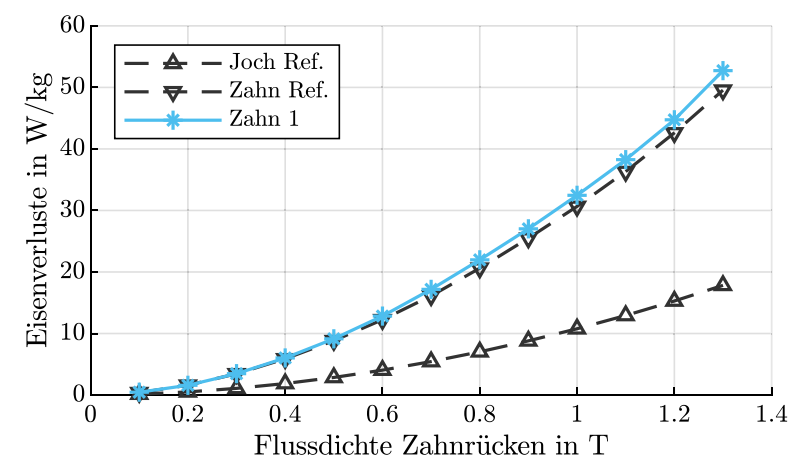

Abb. 11. Auftrennung in Joch- und Zahnverluste bei $0^{\circ}$ Phasenlage und $400 \mathrm{~Hz}$ für Referenzzahn und Zahn 1

sucht werden. Dies bietet zum einen die Möglichkeit optimale Material und Fertigungskombinationen zu ermitteln. Zum anderen können die bestehenden Verlustmodelle mit den gewonnenen Messdaten erweitert und validiert werden.

\section{Konklusion}

Mit dem vorgestellten Messsystem wird die realitätsnahe Bestimmung der Eisenverluste von elektrischen Maschinen mit Einzelzahngeometrie ermöglicht. Durch das implementierte hochdynamische Regelverfahren können die Einzelzähne in allen relevanten Betriebspunkten der Maschine untersucht werden. Dies beinhaltet die flexible Vorgabe von Flussdichteamplitude und Frequenz. Zusätzlich können Harmonische bis zur siebten Ordnung und freier Wahl der Phasenlage eingeprägt werden. Die Grundwelle und die Harmonischen können dabei getrennt für beide Zahnseiten $A$ und $B$ definiert werden. Der Vergleich der simulierten und der gemessenen zeitlichen Verläufe der magnetischen Flüsse hat gezeigt, dass die gewünschten Signalformen mit hoher Genauigkeit eingestellt werden können. Dies ermöglicht die Verlustbestimmung unter anwendungsnahen Feldbedingungen.

Mit dem vorgestellten Verfahren zur Separation der Zahnverluste kann der Fertigungseinfluss auf die Eisenverluste im Einzelzahn dediziert untersucht werden. Unter Zuhilfenahme eines Abbilds des magnetischen Aufbaus in einer 2D-Finite Elemente Simulation, können die Verluste im Einzelzahn von den Verlusten in den Prüfjochen getrennt werden. Die Untersuchung des Modellfehlers hat gezeigt, dass die Verluste mit dem Modellansatz im Betriebsbereich der Maschine mit einem absoluten Fehler kleiner 0,5 W/kg abgebildet werden können. So können die Zahnverluste für alle Betriebspunkte 
der Maschine ermittelt werden. Die Vermessung von Einzelzähnen der gleichen Geometrie aber unterschiedlicher Fertigungsverfahren ermöglicht somit die Charakterisierung des Fertigungseinflusses. In Kombination mit der flexiblen Einstellung der magnetischen Zustände in der Probe können mit dem Einzelzahn-Prüfstand detaillierte Aussagen zu den Eisenverlusten in elektrischen Maschinen getroffen werden.

\section{Danksagung}

Open Access funding provided by Projekt DEAL.

Hinweis des Verlags Der Verlag bleibt in Hinblick auf geografische Zuordnungen und Gebietsbezeichnungen in veröffentlichten Karten und Institutsadressen neutral.

Open Access Dieser Artikel wird unter der Creative Commons Namensnennung 4.0 International Lizenz veröffentlicht, welche die Nutzung, Vervielfältigung, Bearbeitung, Verbreitung und Wiedergabe in jeglichem Medium und Format erlaubt, sofern Sie den/die ursprünglichen Autor(en) und die Quelle ordnungsgemäß nennen, einen Link zur Creative Commons Lizenz beifügen und angeben, ob Änderungen vorgenommen wurden. Die in diesem Artikel enthaltenen Bilder und sonstiges Drittmaterial unterliegen ebenfalls der genannten Creative Commons Lizenz, sofern sich aus der Abbildungslegende nichts anderes ergibt. Sofern das betreffende Material nicht unter der genannten Creative Commons Lizenz steht und die betreffende Handlung nicht nach gesetzlichen Vorschriften erlaubt ist, ist für die oben aufgeführten Weiterverwendungen des Materials die Einwilligung des jeweiligen Rechteinhabers einzuholen. Weitere Details zur Lizenz entnehmen Sie bitte der Lizenzinformation auf http://creativecommons.org/licenses/by/4.0/deed.de.

\section{Literatur}

1. Gmyrek, Z., Cavagnino, A., Ferraris, L. (2012): Estimation of magnetic properties and damaged area width due to punching process: modeling and experimental research. In Proceedings of the XXth international conference on electrical machines (ICEM), Marseille, France 2012 (S. 1301-1308).

2. Hofmann, M., Naumoski, H., Herr, U., Herzog, H.-G. (2016): Magnetic properties of electrical steel sheets in respect of cutting: micromagnetic analysis and macromagnetic modeling. IEEE Trans. Magn., 52(2), 1-14. https://doi.org/10.1109/TMAG.2015. 2484280.

3. Bali, M., Muetze, A. (2019): The degradation depth of non-grain oriented electrical steel sheets of electric machines due to mechanical and laser cutting: a state-of-theart review. IEEE Trans. Ind. Appl., 55(1), 366-375.

\section{Autoren}

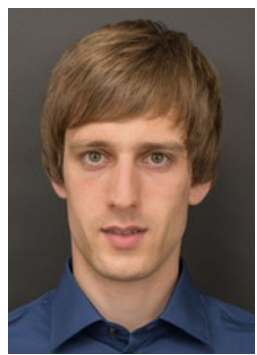

\section{Christoph Rollbühler}

wurde 1989 in Crailsheim, Deutschland, geboren. Seinen Abschluss als Master of Science in Elektrotechnik vom Karlsruher Institut für Technologie hat er 2015 erhalten. Zurzeit arbeitet er am Karlsruher Institut für Technologie an seiner Promotion in Elektrotechnik und Informationstechnologie. Seine Forschungsinteressen umfassen die Modellierung, Parameteridentifizierung und Regelung von Permanentmagneterregten Synchronmaschinen sowie die messtechnische Bestimmung von Kupfer- und Eisenverlusten.
4. Magnetic materials - Part 2: Methods of measurement of the magnetic properties of electrical steel sheet and strip by means of an Epstein frame, IEC 60404-2: 2009-01

5. Magnetic materials - Part 3: Methods of measurement of the magnetic properties of electrical steel strip and sheet by means of a single sheet tester, IEC 60404-3: 201005

6. Bali, M., De Gersem, H., Muetze, A. (2015): Epstein frame measurement based determination of original non-degraded and fully degraded magnetic characteristics of material submitted to laser cutting. In 2015 IEEE energy conversion congress and exposition (ECCE) (S. 6096-6101)

7. Magnetic materials - Part 6 : Methods of measurement of the magnetic properties of magnetically soft metallic and powder materials at frequencies in the range $20 \mathrm{~Hz}$ to $100 \mathrm{kHz}$ by the use of ring specimens, IEC 60404-6: 2018-11.

8. Kahveci, A., Szary, P., Herget, F., Putri, A. K., Hameyer, K. (2016): Methods for hysteresis losses determinations at non-standard ring core geometries equivalent to Epstein measurements. In 2016 th international electric drives production conference (EDPC), Nuremberg, (S. 135-142).

9. Gumbleton-Wood, D., Atkinson, G., Washington, J., Sjöberg, L. (2017): The influence of production methods on the magnetic performance of electrical steels and soft magnetic composites. In 2017 IEEE international electric machines and drives conference (IEMDC) (S. 1-7)

10. Lamprecht, E., Homme, M., Albrecht, T. (2012): Investigations of eddy current losses in laminated cores due to the impact of various stacking processes. In Proceedings of the 2nd international electric drives production conference (EDPC), Nuremberg, Germany (S. 1-8).

11. Clerc, A. J., Muetze, A. (2012): Measurement of stator core magnetic degradation during the manufacturing process. IEEE Trans. Ind. Appl., 48(4), 1344-1352.

12. Breining, P., Kahveci, A., Doppelbauer, M. (2019): Effects of annealing on magnetic properties of laminated stator cores and efficiency of induction machines. In 2019 IEEE international electric machines drives conference (IEMDC) (S. 1038-1044).

13. Veigel, M., Winzer, P., Richter, J., Doppelbauer, M. (2015): New FPGA-based and inline-capable measuring method for the identification of magnetic losses in electrical steel. In Proceedings of international electric drives production conference (EDPC), Nuremberg, Germany (S. 1-6).

14. Veigel, M. (2018): Neues Messverfahren zur Bestimmung der fertigungsabhängigen Eisenverluste von Stator-Einzelzahnblechpaketen in Synchronmaschinen. Dissertation. Karlsruhe: KIT.

15. Axtmann, C., Boxriker, M., Braun, M. (2016): A custom, high-performance real time measurement and control system for arbitrary power electronic systems in academic research and education. In Proceedings of the 18th European conference on power electronics and applications (EPE'16 ECCE Europe), Karlsruhe (S. 1-7).

16. Bertotti, G. (1988): General properties of power losses in soft ferromagnetic materials. EEE Trans. Magn., 24(1), 621-630. https://doi.org/10.1109/20.43994.

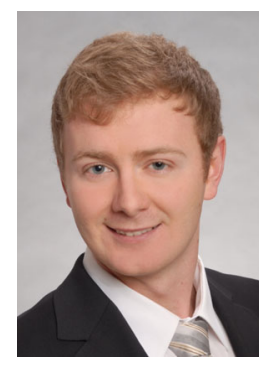

\section{Patrick Breining}

wurde 1989 in Frankenthal (Pfalz), Deutschland, geboren. 2015 erhielt er den Abschluss Master of Science in Elektrotechnik und Informationstechnologie vom Karlsruher Institut (KIT) für Technologie. Seit 2016 ist er am Elektrotechnischen Institut am KIT als wissenschaftlicher Mitarbeiter angestellt. Seinen Forschungsschwerpunkt bilden die messtechnische Bestimmung und Modellierung von Eisenverlusten, sowie die elektromagnetische Auslegung und Optimierung von E-Maschinen für den Traktionseinsatz. 


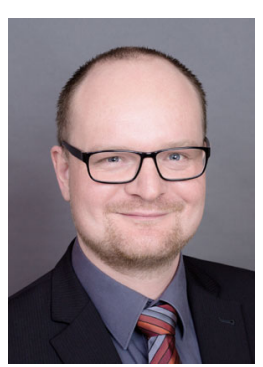

Johannes Kolb

wurde 1982 in Pforzheim, Deutschland, geboren. Seinen Abschluss als Diplomingenieur der Elektrotechnik und Informationstechnik hat er im Jahr 2007 von der Universität Karlsruhe (TH) erhalten. Von 2007 bis 2013 war er am Elektrotechnischen Institut des Karlsruher Instituts für Technologie (KIT) als wissenschaftlicher Mitarbeiter auf dem Gebiet der Leistungselektronik tätig, wo er 2013 seine Promotionsprüfung ablegte. Seitdem leitet er ein Forschungsteam auf dem Gebiet der elektrischen Antriebe und Leistungselektronik bei SHARE am KIT - einer Kooperation der Schaeffler Technologies $A G \&$ Co. KG und dem KIT.

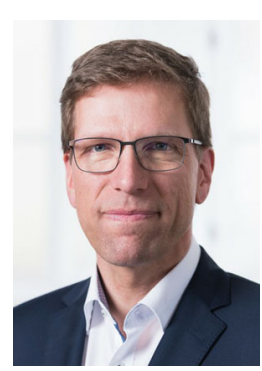

\section{Martin Doppelbauer}

wurde am 26. August 1965 in Altenhundem in Deutschland geboren. Nach dem Studium der Elektrotechnik promovierte er 1995 an der Technischen Universität in Dortmund über ein analytisches Berechnungsverfahren von Wechselstrom-Kommutatormaschinen. Von 1995 bis 2010 arbeitete Martin Doppelbauer in leitender Funktion in der Industrie. Seit 2011 ist Professor Doppelbauer Inhaber der Professur für Hybrid- und Elektrofahrzeuge am Karlsruher Institut für Technologie (KIT). Sein Forschungsinteresse gilt dem elektrischen Antriebsstrang von Fahrzeugen mit besonderem Schwerpunkt auf elektrischen Maschinen mit hoher Leistungsdichte. Prof. Doppelbauer ist auch auf dem Gebiet der Normung tätig und derzeit Vorsitzender des Technischen Komitees 2 (Rotierende Maschinen) der Internationalen Elektrotechnischen Kommission in Genf. 